\title{
Knowledge Sharing and Value Creation in Collaborative Production of Virtual Organization
}

\author{
Caifeng Ma \\ School of Economics and Management Shan Dong JiaoTong University Jinan, China \\ email: cfhorse@163.com
}

\begin{abstract}
Keywords: Knowledge Sharing, Value Creation, Collaborative Production, Virtual Organization, Synergistic Effect.
\end{abstract}

Abstract. We put forward the relationship framework of knowledge sharing and value creating in the virtual organization, based on the analysis of the driving factors and the synergistic effect of knowledge sharing. According to the organization resources, expected return and the knowledge sharing behavior in the virtual organization, we divide the virtual organization into four kinds of collaborative production mode, and analyze synergistic effect of various driving factors of knowledge sharing and value creation in different collaborative production. The results show that the virtual organization members cooperate with for common strategic objectives, organizations achieve knowledge sharing and resources compensation and then cooperative innovation and value creation in the context of trust, respect, equality.

\section{Introduction}

With globalization of economy, progress of information technology, it is difficult for enterprise to make quick response to meet customer change demand, and more and more companies tend to be engaged in sharing knowledge and creating value. Sharing knowledge is the most important factors of production in the knowledge economy (Zack, 1999), is the core of collaborative production network, knowledge sharing is in favor of the integration of knowledge resources of virtual organization, to form a powerful knowledge force, and is beneficial to virtual organization members to realize the interaction and blend on the same platform, to achieve synergistic effect and create value. Researchers have pointed to the importance of knowledge sharing between members within, across, and outside the organization (Chowdhury, 2005) in enhancing the capacity of an organization to innovate and produce quality solutions quickly (Daellenbach \& Davenport, 2004). As a result, many partner firms in a virtual organization collaborate with their suppliers and customers and even competitors, and share information and knowledge, aiming to create a collaborative network that is capable of competing effectively in the marketplace (Ketikidis et al. 2008, Koh et al. 2008). In this study, we discussed the driving factors and synergy effect of knowledge sharing in virtual organization; expect to find that trust and organizational culture and resource/capability are important antecedents of knowledge sharing between many partners, knowledge sharing facilitate cooperative innovation and value creation, thereby improving creative performance..

\section{Collaborative Production Mode in Virtual Organization}

With the change of business environment, organizations constantly seek ways to facilitate and enhance creative, innovative behaviors, and in gaining and sustaining a competitive edge in today's marketplace, more and more enterprises rapidly establish virtual organizations to achieve cooperation and competition advantages through resource sharing and complementary between partners, improve the efficiency of cooperative innovation, promote the performance of the organization.

Goldman et al (1991) put forward the concept of virtual enterprise for the first time, in order to grasp the largest market adaptability, virtual enterprise is more rapid formation of the alliance, temporary company or department to grasp the fast-changing market opportunity. John A. Byrne (1993) argues that "virtual enterprise" are independent of enterprise, each other operators, including 
suppliers, customers and competitors, the temporary enterprise network through connect information technology, to share the cost and the related skills and knowledge resources, joint development and entering new markets; It has no organization chart, there is no central office; Neither vertical integration, nor have any level.

There are three collaborative production modes in virtual organizations, the first is supply chain outsourcing model, such as dell, Nike, is focusing on the one-way mode, lock in a technology and standards of partner; Followed by open source collaboration outsourcing mode, such as the Boeing 787 collaborative research and development, is a two-way interaction between Boeing and its suppliers partners. Boeing open source technology, sharing cooperate achievement with partners; the third is the crowdsourcing model, i.e., mass collaboration model, such as creative bazaar, Alibaba creating business ecosystem (Si JianBo, 2009). In broad terms, virtual organization includes individual form self-organization based on a common goal, and organizations establish cooperation alliance and technical exchanges in order to achieve a certain technical innovation strategic target. we study the generalized virtual organization, include four organizations, for example, the supply chain outsourcing, value chain cooperation, Crowd sourcing, and Wiki, on the basis of the resources, expected return and knowledge sharing behavior in the virtual organization, outsourcing and value chain cooperation are cooperation alliance that consisted of more than two organizations. The latter two belong to the individual and the organization's open collaborative production mode, individual voluntary attend.

Outsourcing is the core enterprise in order to maintain organizational competitive ability, and lack of organization human predicament, entrusted non-core business to external professional company, to reduce operating costs, improve quality, focus on human resources, and improve customer satisfaction. Value chain cooperation is two or more enterprises or multinational companies in order to achieve common strategic target and make mutual cooperation, take total risk, share interests' joint operations. Crowdsourcing is a kind of brand-new business model that the enterprises through the Internet tools distribute work outside the enterprise in order to find creative idea or solve technical problem, i.e. a company or organization outsourcing work tasks in the past by employees perform, in the form of free voluntary approach to the non-specific public networks. The Wiki model is the new ways of producing the products and service by mass collaboration, operating core is Openness, Peering, Sharing and Acting Globally.

\section{The Driving Factors of Knowledge Sharing in Different Cooperation Production of Virtual Organization}

There are two kinds of human knowledge, namely explicit knowledge and tacit knowledge, explicit knowledge can be stated written text, charts, and mathematical formula, tacit knowledge can only needs not talk (Polanyi, 1958; Nonaka, 1958).Cummings and Teng (2003) suggests that the more difficult expressive of knowledge, the more difficult for sharing knowledge. Davenport and Prusak think possession of knowledge will benefit more than sharing knowledge, knowledge owners may not participate in the operation of knowledge market, become a supplier of knowledge. Because the employees are rational, to monopolize the desire of knowledge, and is not willing to take the initiative to share their tacit knowledge, therefore, enterprises must establish a kind of incentive mechanism, so as to promote the employee's knowledge sharing. Trust mechanism is the basic incentive mechanism of enterprise knowledge sharing network.

Trust. The existing literature shows the trust is important in business transactions, operation effect, the quality of the products (Shin et al. 2000) and the relationship between the effect of the overall interests(Szulanski et al. 2004), trust reduced monitoring costs associated with knowledge sharing (Williamson1996); Trust and relationship commitment can promote cooperation relations (e.g. Ring and Van de Ven 1994, Kumar et al., 1995), improve the cooperation performance, promote the exchange of knowledge (e.g. Dyer and Chu 2003, directed et al., 2005, Shun Cai, Mark Goh, Robert DE Souza, 2013) .In the case of a trust, knowledge sharing intention (Kaufman et al., 2000, 2008 
Renal, Cai et al., 2010) and the amount of information can be exchanged (Dirks and Ferrin 2001, Szulanski et al., 2004) increase, and increase the cooperation between partners.

There are both explicit knowledge sharing and tacit knowledge sharing in cooperative alliance. in the presence of trust, knowledge providers are more willing to provide useful knowledge, knowledge receivers are more willing to absorb and assimilate knowledge that provider knowledge transfer (Andrews and Delahay 2000).Trust, therefore, help to overcome the obstacle of knowledge sharing, and promote knowledge exchange, especially technical knowledge sharing among partners in various situations (Andrews and Delahay 2000). Trust is not only a trading lubricant, and it is the core of the deal. Trust in knowledge sharing is more important than the formal cooperation program, because if there is no trust, knowledge sharing is not possible (Andrews \& Delahay, 2000). Therefore, we think that the trust between members across enterprises is the basis of the successful knowledge sharing in cooperative alliance.

Crowd sourcing and Wiki model, the two open collaboration network organization are the organization structure supported by the trust, the trust is seen as the important variable to the development of the network organization. Trust connection between all parties, interstitial trust is one of decisive factors to realize network organization target, the lack of trust will lead to the failure of the cooperation. Therefore, trust is the basis of the formation and development of the open collaborative production in network organization.

Expected Return. Define Cooperation alliance is formed based on the expected return of organization, for example the union will be able to face various aspects of globalization challenges such as the market, products, technology innovation and so on; Alliance can develop stronger complementary advantages, reduce the cost and risk, to form a win-win situation, partners is a community of interests, value sharing is the drive factor of knowledge sharing in cooperation alliance.

The task of Crowd sourcing and wiki are usually borne by the individual in open collaborative productions of virtual organization, individual is active for knowledge sharing. Based on social cognitive theory, the result is expected on the expected results of self behavior, individual behavior will lead to positive results, because individual expectations are met, he will implement knowledge sharing. Results expectations are the important factors that affect the knowledge sharing decision. On the one hand, the results show that if participators think that they'll get inherent interests such as monetary rewards, promotion or re-education opportunities, they will form a positive attitude toward knowledge sharing; On the other hand the participators think that they will get the external benefits such as self satisfaction, social identity, or rights, they will also be willing to knowledge sharing. The individual will to act according to principles of rational self-interest based on the principles of economic exchange. Therefore, when the result of the knowledge sharing more than the cost or reaches the expected value, the knowledge sharing will happen.

Organizational Culture. Ruggles (1998) surveyed the business manager and found that organizational culture is the biggest barrier to knowledge transfer and knowledge sharing. Wu qiang, zhang weiguo (2014) argue that organization culture and atmosphere affects the success of knowledge sharing. He jin (2005) carried on the empirical research on incentive mechanism, it is concluded that intrinsic motivation factors, knowledge sharing "force" (represented by the knowledge sharing atmosphere) and "leadership and institutional factors" has a positive effect on knowledge sharing activities. Herzberg (1968) argue that, only incentive factors can constitute a knowledge provider of knowledge sharing motivation, mainly including a sense of achievement, responsibility, recognition of work, the opportunity to be in an important position, and challenging work. Many studies have shown that knowledge sharing is more effective in a proper incentive culture atmosphere (Xiaoming Huang, 2006; Yonghua Fu and Jiuchang Zhou, 2008; O 'Dell and Grayson, 1998; Herzberg, 1968). The premise of Alliance success is that every partner fully respects and trusts of the company's brand and enterprise culture to ensure the independent status of each side. Innovation is derived from the diversified, accept diversified value, diversification can support to innovation. Therefore, in cooperation between alliance members, partners fully respect their own corporate culture, and form a cooperative atmosphere of mutual trust, to ensure the smooth progress of knowledge sharing. 
Resource/Capability. No organization is self-sufficient. Company always depends on their trading partners. Prior research has shown that power is an important predictor of channel relationship (Kumar et al. 1998), firm profits in supply chains (Cool and Henderson, 1998), and customer integration (Zhao et al. 2008).Resource dependence theory asserts that inter-organizational dependence is created when one business partner does not entirely control all necessary conditions to complete an action or obtain a desired outcome (Pfeffer and Salancik 197840 pages). The power structure between business partners is highly correlated with the degree of interdependence and its balance, which is determined by who has the control of key resources. Knowledge sharing between business partners require that business practices, information, and technology know-how be shared. A firm becomes more powerful to its partner if it controls some resources necessary and important to the partner, and vice versa. The greater the bargaining power a firm has over its business partners, the greater the likelihood it can obtain resources from its business partners. Thus, we suggest that the asymmetric control of key resources between the firm and its business partners will significantly affect the degree of knowledge sharing between them. Then a scientific contract must specify responsibility and moral duty of knowledge sharing between alliance partners.

\section{KNOWLEDGE SHARING AND VALUE CREATION IN DIFFERENT COOPERATION PRODUCTION OF VIRTUAL ORGANIZATION}

Knowledge Sharing and Value Creation in Cooperative Alliance. Cooperative Alliance is a kind of strategy of swaps and resource sharing and integration of cooperative parties. Better utilization of resources for the enterprise is to save a lot of expenses, and to get more effect. Enterprises management strength and resources are the same in value chain cooperation, so the knowledge flow between enterprises is a two-way street. Both sides provide their own expertise, a common development of new technology; coordinate with each other, so as to achieve innovation. Outsourcing companies pass the company's technology and knowledge to partners by sharing knowledge; by sharing all kinds of demand information, suppliers can reduce or eliminate the related cost and management cost, and all parties obtain greater benefits. Resources complementary, alliance contracts, trust, respect and expected return, will promote the coordination effect in virtual organization, and reduce the management risk and transaction costs, and realize the value of appreciation of the organization, to achieve a win-win situation (see Fig 1).

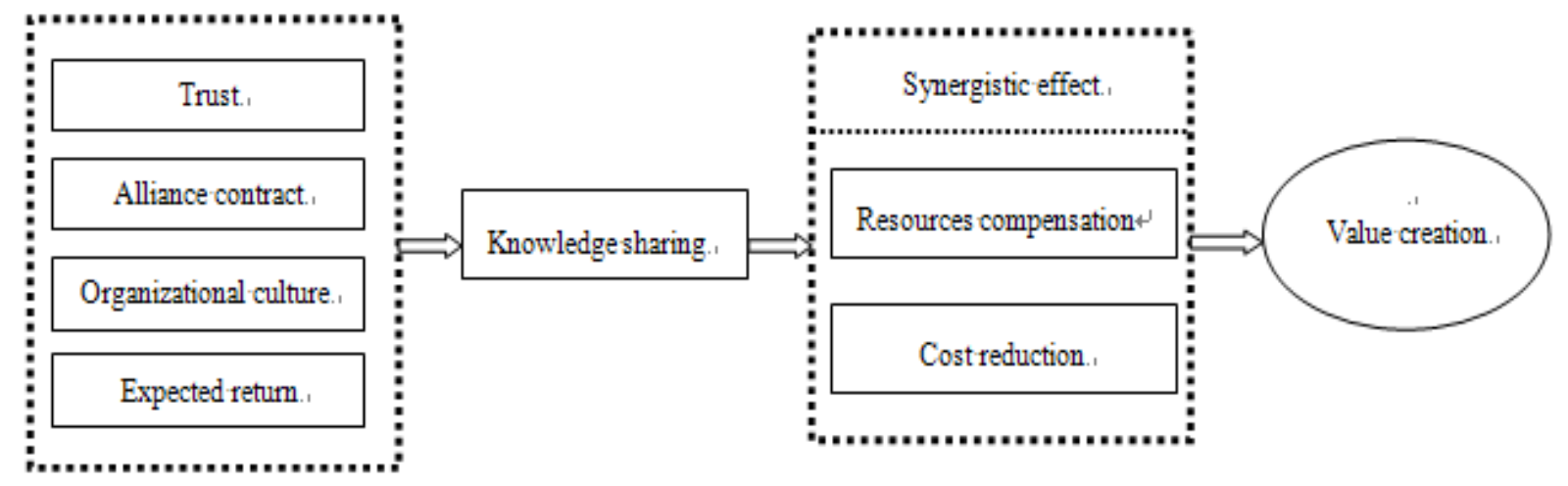

Figure 1 Knowledge sharing and value creation in cooperative alliance.

Knowledge Sharing and Value Creation in Open Collaborative Production. The enterprises and individuals can find cooperation partners and acquire technology innovation, with small transaction costs can be identified as infinite close to zero, after making use of Crowd sourcing and Wiki mode. It can reduce transaction costs, speed up the pace of innovation, and make all participants in the markets more efficient; customers will get better services and products due to a lower price. Participator in open collaborative production make personal expertise interactive sharing, and obtained the certain material benefit, and got the spirit incentives, such as high achievement. Crowd sourcing 
and Wiki modes are two types of knowledge sharing, and speed up the cooperative innovation and knowledge creation, to realize win-win of customers, enterprise and participator (as shown in Fig 2).

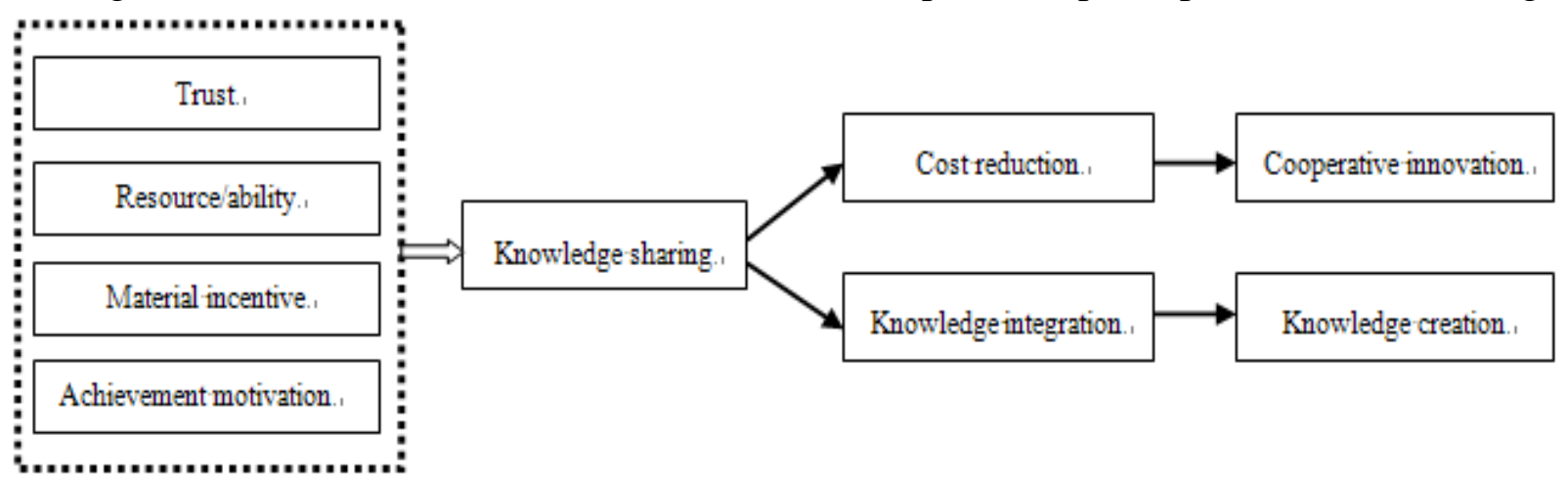

Figure 2 Knowledge sharing and value creation in open collaborative production.

\section{Conclusions}

We analyze the driving factor of knowledge sharing and cooperation value creation in different virtual organization. We find that the firm cooperates with partners, shared sacrifice and shared values for the common strategic target in the virtual organization, under supply chain outsourcing and the value cooperation. Partners share knowledge with partners in the context of trust, respect, and fair and reasonable coordination production and realize the significant synergies, achieve resources complement and effective value creation, achieving the overall goal of organization, then:

Proposition 1: trust, respect their own culture, reasonable return and scientific alliance contract are the main driving factors of knowledge sharing in cooperative alliance;

Proposition 2: enterprises achieve complementary resource and cost reduction and effective value creation as a result of knowledge sharing between cooperative partners.

In the open collaborative production (Crowdsourcing and Wiki mode), the enterprise obtain needed services, ideas, or content by soliciting contributions from a large group of people who are voluntary for knowledge sharing, and can obtain higher efficiency of sharing. The organization attract talent from outside organization and create value with the user and the talent from outside organization, to make them to participate in the innovation and the cooperation process of the enterprise, reduce the cost and risk, improve efficiency. Participants are more reciprocity motivation, lower material incentives or unpaid participation, they focus on the sense of accomplishment and participation, and pay attention to play to their expertise in an atmosphere of trust, equality, finally achieves the cooperative innovation and knowledge creation, then:

Proposition 3: trust, ability, the desired material rewards and a sense of accomplishment is the main driving factors of knowledge sharing in open collaborative production;

Proposition 4: Enterprises achieve cost reduction, innovation and knowledge creation as a result of sharing knowledge from participants in open collaboration production.

Our study also provides some managerial implications for organization managers. Organization should encourage knowledge sharing with their partners, because ultimately knowledge sharing would benefit both parties. Organization emphasize on different knowledge sharing under different situations and should take corresponding measures to strengthen the cultural atmosphere of the organization, make knowledge sharing cooperation, innovation and value creation in the atmosphere of trust, respect. Organization should improve the credibility of Internet users, increase the participants' expectations (the appropriate material incentive and spiritual incentive), these are the foundation of the survival and development in open collaborative production in virtual organizations. 


\section{Acknowledgements}

This work was financially supported by the Shandong Social Science Foundation (16CGLJ35, 16CKJJ26), Education and Research Fund subject of Shandong Jiaotong University (Jy201609).

\section{References}

[1] Lee J N. 2001.The Impact of Knowledge Sharing, Organizational Capability and Partnership Quality on IS Outsourcing Success. Information \& Management 38:323-335

[2] Connelly C E, Kelloway E K. 2003. Predictors of Employee' Perceptions of Knowledge Sharing Cultures. Leadership \& Organization Development Journal 24:294 301.

[3] Shun Cai, Mark Goh, Robert de Souza. 2013. Knowledge sharing in collaborative supply chains: twin effects of trust and power. International Journal of Production Research 51:2060-2076.

[4] Andrews KM, Delahaye BL.2000.Influence on Knowledge Processes in Organizational Learning: The psychosocial filter. Journal of Management Studies 37:797-810.

[5] Zhao, X., et al. 2008. The impact of power and relationship commitment on the integration between manufacturers and customers in a supply chain. Journal of Operations Management 26:368-388.

[6] Hamel G. 1991.Competition for Competence and Inter-Partner Learning Within International Strategic Alliance. Strategic Management Journal 12:83-103.

[7] Williams, T.2005.Cooperation by design: structure and cooperation in international networks. Journal of Business Research58:223-231

[8] Ikujiro Nonaka. 1991. The Knowledge-Creating Company. Harvard Business Review 69:96-104.

[9] Cummings J L, Teng B S. 2003. Transferring R\&D Knowledge: the Key Factors Affecting Knowledge Transfer Success. Journal of Engineering and Technology Management 20:39-68.

[10] Dyer, A. J. and Singh, H. 1998. The Relational View: Cooperative Strategy and Source of Interorganizational Competitive Advantage. Academy of Management Review 23: 660-679.

[11] Jianbo Si. 2009. Nature and cause of the virtual organization. Jilin University.

[12] Abraham Carmeli, Roy Gelbard, Roni Reiter-Palmon.2013. Leadership, Creative Problem-Solving Capacity, and Creative Performance: The Importance of Knowledge Sharing Human Resource Management 52:95-122. 